\title{
Air-spaced GaN nanopillar photonic band gap structures patterned by nanosphere lithography
}

\author{
K. H. Li and H. W. Choi ${ }^{\text {a) }}$ \\ Department of Electrical and Electronic Engineering, The University of Hong Kong, Pokfulam Road, \\ Hong Kong
}

(Received 26 July 2010; accepted 27 November 2010; published online 19 January 2011)

\begin{abstract}
We report on the fabrication of ordered hexagonal arrays of air-spaced $\mathrm{GaN}$ nanopillars by nanosphere lithography. A self-assembled two-dimensional silica nanosphere mask was initially formed by spin-coating. Prior to pattern transfer to the GaN substrate, a silica-selective dry etch recipe was employed to reduce the dimensions of the nanospheres, without shifting their equilibrium positions. This process step was crucial to be formation of air-spaced hexagonal arrays of nanospheres, as opposed to closed-packed arrays normally achieved by nanosphere lithography. This pattern is then transferred to the wafer to form air-spaced nanopillars. By introducing air gaps between pillars, a photonic band gap (PBG) in the visible region can be opened up, which is usually nonexistent in closed-packed nanopillar arrays. The PBG structures were designed using the plane wave expansion algorithm for band structure computations. The existence and positions of band gaps have been verified through optical transmittance spectroscopy, which correlated well with predictions from simulations. From photoluminescence (PL) spectroscopy, a fourfold increase in PL intensity was observed and compared to an as-grown sample, demonstrating the effectiveness of well-designed self-assembled PBG structures for suppressing undesired optical guiding mode via PBG and for promoting light extraction. The effects of defects in the nanopillar array on the optical properties are also critically assessed. () 2011 American Institute of Physics.
\end{abstract}

[doi:10.1063/1.3531972]

\section{INTRODUCTION}

Lateral photon guiding is one of the major issues concerning gallium nitride $(\mathrm{GaN})$ light-emitting diodes (LEDs), caused by the stark contrast in refractive index between the semiconductor layer with its ambient, resulting in restricted light extraction and subsequent reabsorption. Surface texturing ${ }^{1}$ techniques can be adopted to alleviate this problem, with different degrees of success depending on the dimensions, ${ }^{2}$ geometries, ${ }^{3}$ and regularities of patterns ${ }^{4}$ employed. In general, microstructures involve simple microfabrication processes, exposing additional surface areas for light extraction. ${ }^{5}$ On the other hand, ordered nanostructures such as photonic crystals $(\mathrm{PhCs}),{ }^{6-8}$ typically rely on electronbeam or nanoimprint lithography ${ }^{9}$ for patterning, the cost and efficiency of which is often forbidding. Nevertheless, $\mathrm{PhCs}$ are highly effective, possessing the ability of controlling spontaneous emission in optoelectronic devices. The formation of photonic band gap (PBG) structures inhibits all wave vectors within the $\mathrm{PBG},{ }^{10}$ and thus promotes light extraction by diffracting waveguided modes out with the semiconductor, as illustrated in Fig. 1.

Conventionally, $\mathrm{PhC}$ are patterned as ordered arrays of recessed air-holes or protruding pillars. Instead of employing high-cost direct-write techniques, nanosphere lithography (NSL) (Ref. 11) is adopted in this work, being a practical alternative approach toward large-scale nanofabrication and with the capability of forming two-dimensional and threedimensional (3D) $\mathrm{PhC}$ structures. Uniform spheres are ca-

${ }^{a)}$ Electronic mail: hwchoi@hku.hk. pable of self-assembling into ordered hexagonal arrays over large areas; such monolayers of nanospheres serve perfectly as hard masks to transfer pillar patterns onto the surfaces of LEDs. The ability of spheres to spontaneously form closedpacked structures also acts as its limitation; PhCs require alternating layers of different materials with defined and constant separations, and a close-packed structure is obviously not favorable for achieving this. Establishing finite spacing between individual spheres is a critical step toward realizing PBG structures.

In this paper, we propose an air-spaced nanopillar $\mathrm{PhC}$ structure with a wavelength-tunable PBG. Prior to pattern transfer to the wafer, silica nanospheres are shrunk by a selective dry etch process. As a result, spacing is induced between spheres on the plane without altering the sites of spheres; therefore the packing of the modified array remain largely regular. This dimension-adjusting procedure overcomes the restrictions of close-packed patterning to achieve
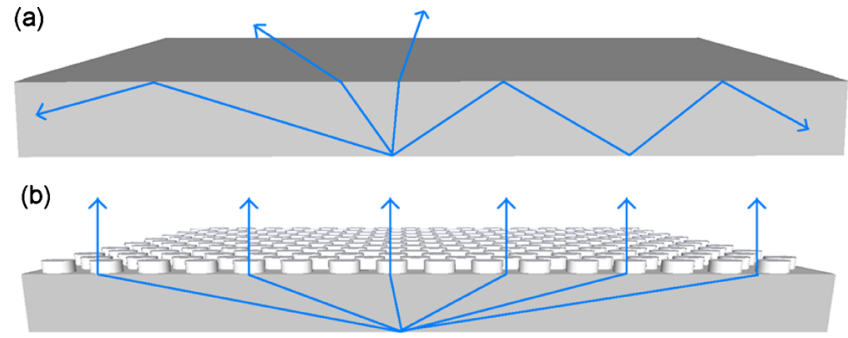

FIG. 1. (Color online) (a) A large fraction of the light is trapped within the semiconductor layer due to total internal reflections. (b) PBG structures promote light extraction by diffracting waveguided modes out of the layer. 


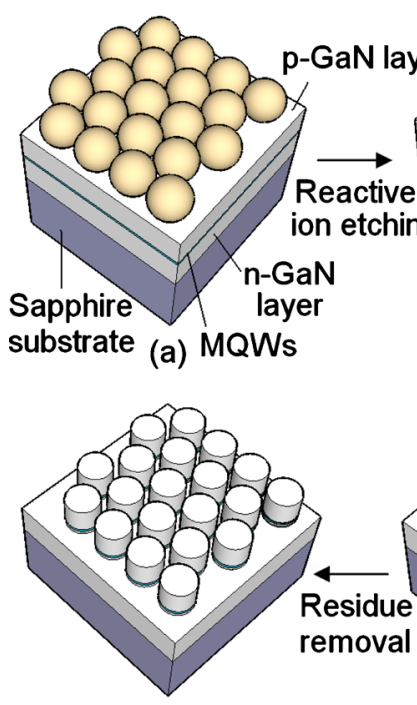

(d)

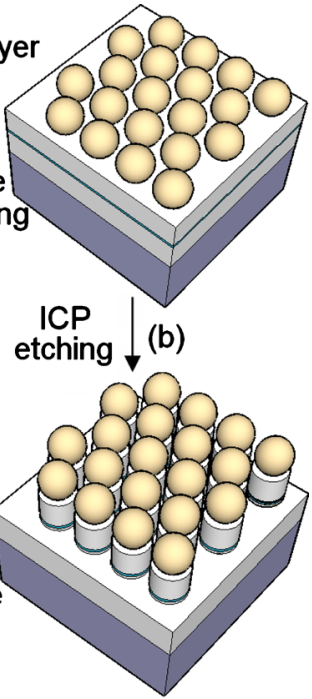

(c)
FIG. 2. (Color online) Schematic diagrams illustrating the fabrication flow (a) Silica nanospheres are coated onto the surface of a GaN wafer, forming closed-packed arrays. (b) Shrinkage of spheres using RIE. (c) Pattern transfer to $\mathrm{GaN}$ using ICP etching. (d) Silica residue removal.

low-cost, high-efficiency, and PBG-tunable nanopillar arrays, which has been applied to InGaN LED materials for realizing enhancement of light extraction.

\section{EXPERIMENTAL DETAILS}

Figure 2 illustrates the proposed fabrication process of air-spaced nanopillar arrays by NSL. Our hybrid NSL process was performed on III-nitride LED wafers consisting of InGaN/GaN multi-quamtum wells (MQWs) grown by metalorganic chemical vapor deposition on c-plane sapphire substrate. Uniform silica $\left(\mathrm{SiO}_{2}\right)$ nanospheres, suspended in deionized water with mean diameters of $192 \mathrm{~nm}$, were mixed with sodium dodecyl sulfate (SDS) at a volume ratio of 10:1. SDS acted as a surfactant to reduce the surface tension of water and thus facilitated the spreading of particles to prevent clustering. $1.5 \mu \mathrm{L}$ of well-mixed colloidal suspension was then dispensed onto a sample surface by mechanical micropipetting. The nanospheres spread laterally upon spincoating at $1000 \mathrm{rpm}$ for $5 \mathrm{~min}$, self-assembling into a monolayer hexagonal-close-packed array across the sample. At this time, the pattern can be transferred to the LED wafer to form closed-packed nanopillars, as demonstrated from our previous works. ${ }^{12,13}$ In this work, the nanosphere-coated wafer was subjected to reactive ion etching (RIE) using $\mathrm{CHF}_{3}$-based plasmas at low rf power prior to pattern transfer. This choice of etchant gas ensured that the silica spheres are selectively etched, without affecting the GaN substrate. During the etching process, the dimensions of the silica nanospheres are reduced. The rf power was maintained low in order to avoid overheating (causing distortion of sphere geometry), and translation of spheres (destroying orderliness of packing). Due to dimensional reduction, an air-gap is induced between spheres. The shrunk nanospheres then served as an etch mask and the pattern was subsequently transferred to the GaN wafer by inductive-coupled plasma (ICP) etching using $\mathrm{Cl}_{2}$ chemistry to form an air-spaced nitride nanopillar

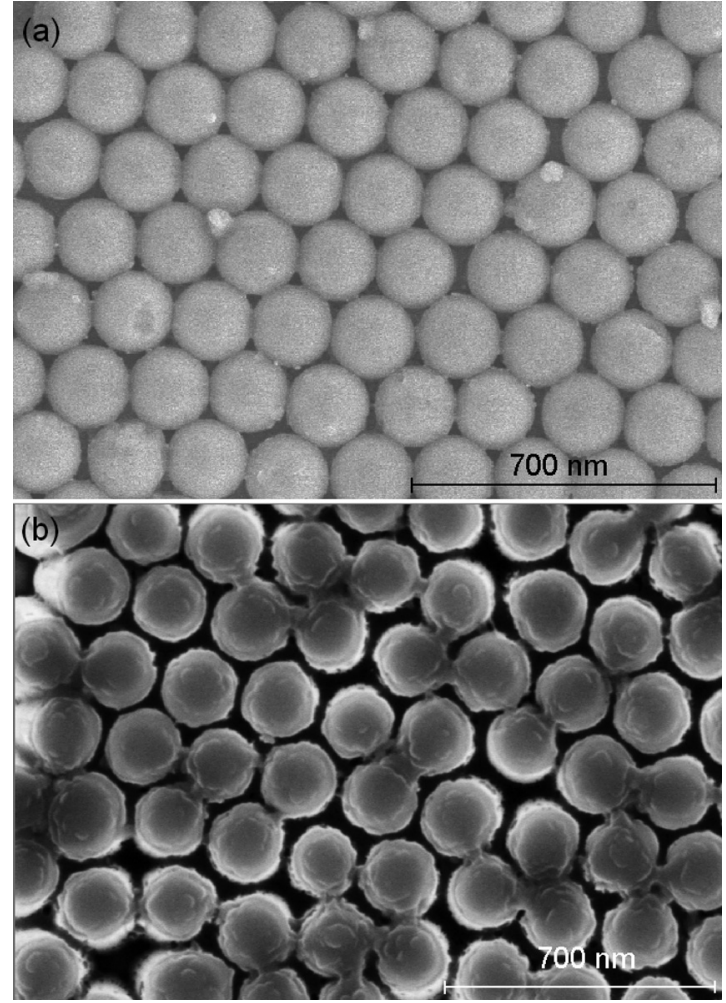

FIG. 3. FE-SEM images showing (a) the original ordered close-packed nanopillar array and (b) the air-spaced nanopillars.

array. The ICP and platen powers were maintained at 300 and $100 \mathrm{~W}$ while the chamber pressure was fixed at $5 \mathrm{mTorr}$. The etch depth was approximately $350 \mathrm{~nm}$ after etching for $75 \mathrm{~s}$. The spacing between nanopillars was exposed as imaged by field-emission scanning electron microscopy (FESEM) in Fig. 3. The nanospheres were subsequently removed by sonification in de-ionized water, leaving behind the nitride air-spaced nanopillar array.

Optical transmittance measurements were conducted to verify the existence and position of a PBG. The incident beam from a high power broadband solid-state plasma light source (Thorlabs HPLS-30-03) was collected by an optical fiber and focused onto the samples in planar direction. The transmitted beam was collected and channeled to an optical spectrometer (Ocean Optics HR2000) via another fiber. The optical properties of the PhC structures were further evaluated by time-integrated photoluminescence (PL) at room temperature. A Spectra-Physics diode-pumped solid-state UV laser at $349 \mathrm{~nm}$ was used as an excitation source $(120 \mu$, $1 \mathrm{kHz}$ ) while the PL signal was coupled to a spectrometer comprising an Acton SP2500A $500 \mathrm{~mm}$ spectrograph and a Princeton Instrument PIXIS open-electrode charge-coupled device via an optical fiber bundle, which offers optical resolutions of better than $0.1 \mathrm{~nm}$.

\section{RESULTS AND DISCUSSIONS}

\section{A. Designing the PhC structure}

To design and predict the existence of a PBG in a nitride nanopillar array, band diagrams were computed using RSOFT BANDSOLVE, which employs the plane wave expansion 


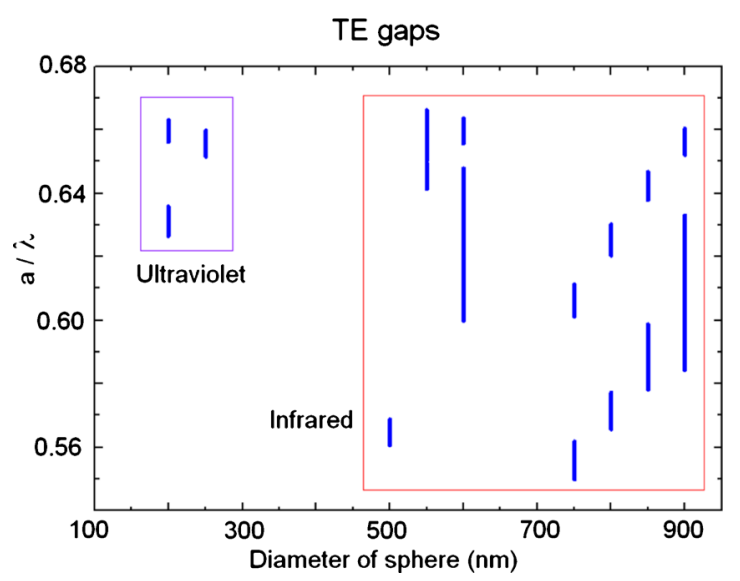

FIG. 4. (Color online) Plot of simulated TE PBG as a function of diameters of close-packed spheres.

(PWE) algorithm for band computations. The supercell technique was utilized during 3D PWE simulations. We begin with the simulation of a closed-packed nanopillar array, the structure obtained naturally with NSL. In this case, the periodicity of the array is considered to be the diameter of a single nanosphere. The positions of the PBG $(a / \lambda)$ for the transverse electric (TE) modes were computed as a function of sphere diameter (between 100 and $900 \mathrm{~nm}$ ) and plotted in Fig. 4, since emissions from InGaN/GaN MQWs are dominated by TE modes. ${ }^{14}$ The plot shows that the TE-PBGs are mainly located in the ultraviolet and infrared regions of the spectrum. For visible InGaN/GaN LED applications, the PBG should obviously be located within the visible spectrum to achieve any beneficial effects. Of course, even in the absence of a PBG, the closed-packed nanopillar structure can still be used to increase light extraction via dispersive and geometrical effects, ${ }^{15}$ albeit with reduced effectiveness.

In spite of the said limitations, a PBG can still be introduced into the visible spectral region by modifying the NSL process to enlarge the physical gaps between nanopillars to produced air-spaced nanopillar arrays, as described in the section on experimental details. To demonstrate this concept, our experiments and computations were carried out using nanospheres with initial nominal diameters of $192 \mathrm{~nm}$ with variations of $+/-2 \mathrm{~nm}$. The positions of the TE band gaps for shrunk nanopillar arrays of diameters 120-170 nm, with a fixed pitch of $192 \mathrm{~nm}$, were computed and shown in Fig. 5. By reducing the diameters of spheres while maintaining their pitch, the positions of the TE-PBG shift accordingly. As we are interested in the visible region, the range of frequencies $(\mathrm{a} / \lambda)$ of interest lies between 0.39 and 0.45 , corresponding to wavelengths of between 426 and $492 \mathrm{~nm}$. From the simulation results in Fig. 5, it can be deduced that the diameters of spheres should be reduced into the range of 126-162 nm, coinciding with the range of $\mathrm{InGaN} / \mathrm{GaN}$ MQWs emission centered at $455 \mathrm{~nm}$ for the material used in our experiments.

\section{B. Fabrication and optical characterization of the PhC structure}

The proposed air-spaced nanopillar structure was fabricated using a modified NSL process. The self-assembled

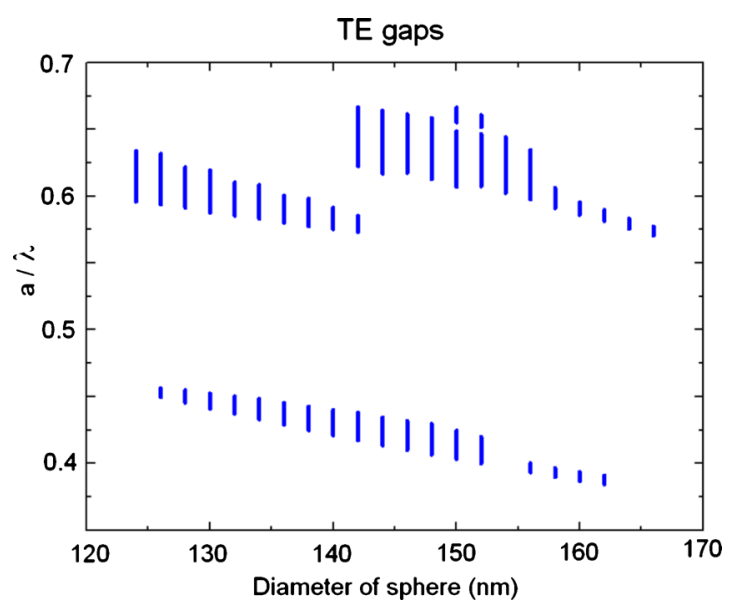

FIG. 5. (Color online) Plot of simulated TE PBG as a function of diameter of sphere (with a pitch of $192 \mathrm{~nm}$ ).

spheres, which acted as a sacrificial masking layer, were etched for dimensional reduction. During this first etching step, the silica spheres are etched without pattern transfer to the $\mathrm{GaN}$ wafer. As the dry etch process is directional, the rate of etching in the vertical direction is faster than in the lateral plane. Gradually the incident ions trim the diameters of the spheres, opening up air gaps between spheres. However, under prolonged etching, the geometry of spheres may become irregular in shape and adjacent spheres tend to aggregate, resulting in disruption of order in the arrays, due to physical bombardment of ions and accumulation of heat. Figures 6(a) and 6(b) shows FE-SEM images of nanosphere arrays after

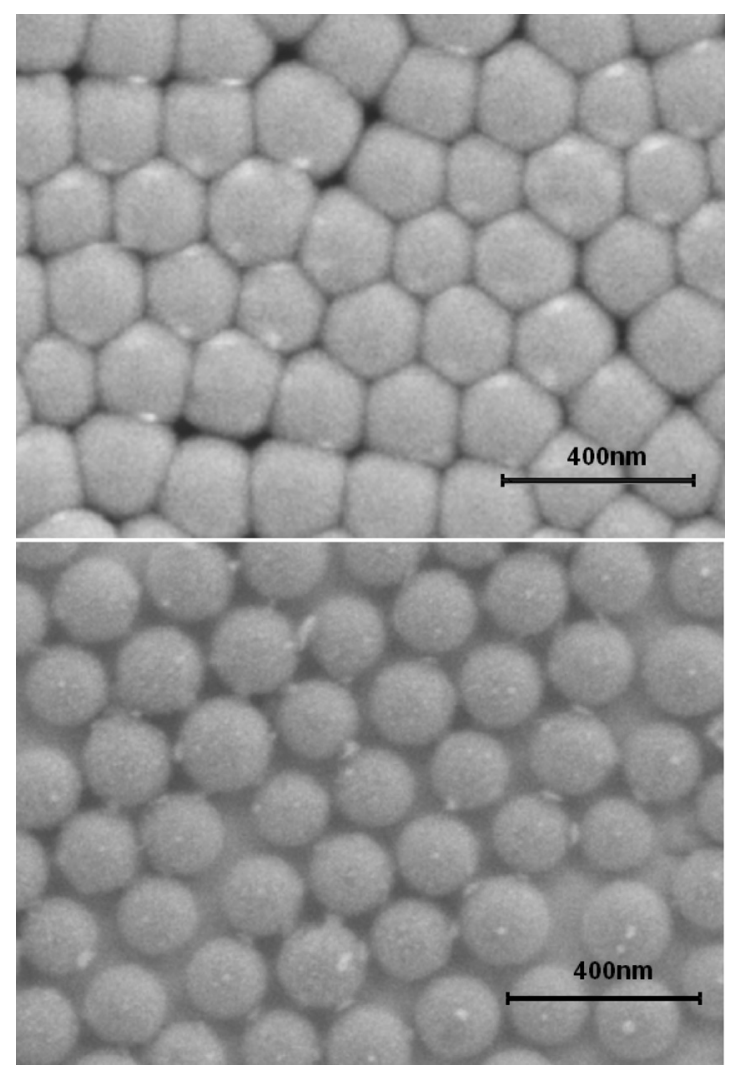

FIG. 6. FE-SEM images showing nanosphere arrays after dry etching at (a) 300 and (b) $70 \mathrm{~W}$ rf powers. 

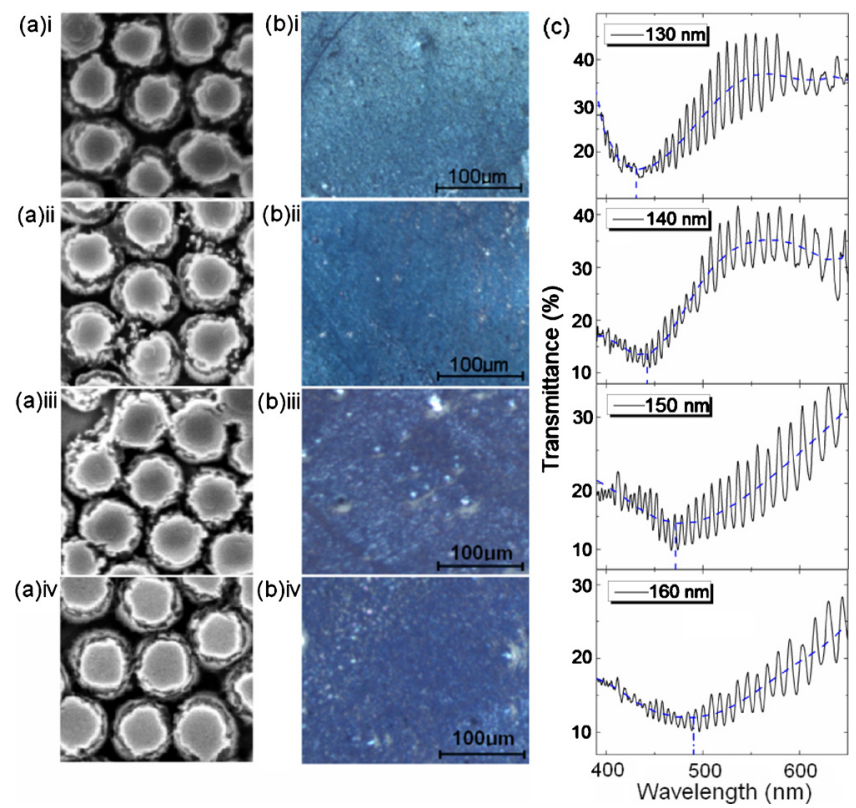

FIG. 7. (Color online) (a) FE-SEM images showing air-spaced nanopillar arrays that have been RIE etched for durations of (i) 14, (ii) 12, (iii) 10, and (iv) $8 \mathrm{~min}$; (b) optical microphotographs of nanopillar arrays with diameters of (i) 130, (ii) 140, (iii) 150, (iv) and $160 \mathrm{~nm}$, and (c) measured transmission spectra from the respective nanopillar arrays.

etching at $300 \mathrm{~W}$ and $70 \mathrm{~W}$ of $\mathrm{rf}$ powers, respectively. To produce nanopillar arrays of desired order and uniformity, moderate etch conditions are required to minimize defect formation. Based on our calculations, four sets of samples were developed with incremental etch durations. After pattern transfer to the $\mathrm{GaN}$ wafer and residue removal, four samples with air-spaced nanopillar structures were produced. The FESEM images in Fig. 7(a), i-iv, illustrate nanopillar arrays etched for durations of $14 \mathrm{~min}, 12 \mathrm{~min}, 10 \mathrm{~min}$, and $8 \mathrm{~min}$, respectively; their diameters are roughly equal to $130 \mathrm{~nm}$, $140 \mathrm{~nm}, 150 \mathrm{~nm}$, and $160 \mathrm{~nm}$, respectively.

Plan view microphotographs in Fig. 7(b), i-iv, illustrate physical color changes observed from the sample surface in the normal direction, changing from purplish blue to greenish blue with decreasing pillar diameter. Light spots are attributed to larger area of defects which are unpatterned. To verify the existence and position of the PBG, an optical transmission measurement in the planar direction was conducted. Figure 7(c), i-iv, shows the measured transmission spectra. Four distinct transmission minimize were observed at the wavelengths of $490.59 \mathrm{~nm}, 471.49 \mathrm{~nm}, 444.51 \mathrm{~nm}$, and $431.96 \mathrm{~nm}$, respectively, with decreasing sphere diameters of 160, 150, 140, and $130 \mathrm{~nm}$. As the PBG structure forbids lateral propagation at the range of wavelengths within the band gap, propagation of the incident beam along the plane is restricted, giving rise to reduced transmission at those wavelengths. For light with wavelengths beyond the band gap region, laterally propagating photons do not experience PBG confinement effects. The transmission spectra, which indicate the PBG positions, correlated well with the PWE stimulated results in Fig. 5.

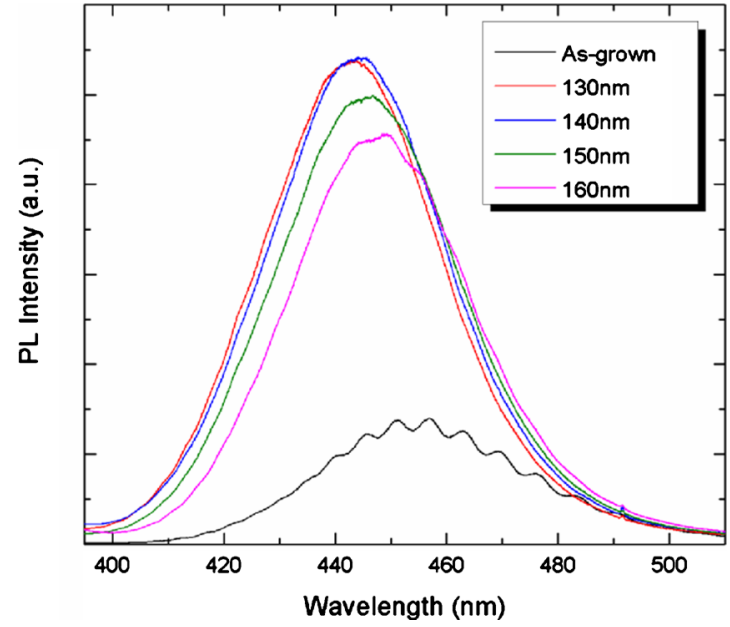

FIG. 8. (Color online) Measured PL spectrum from air-spaced nanopillar arrays, compared with an as-grown sample.

\section{Enhancement of PL intensity in PhC structures}

The optical effects of incorporating these $\mathrm{PhC}$ structures onto LED wafers were evaluated by PL measurement. Figure 8 shows measured PL spectrum from the four nanopillar arrays, together with the PL spectrum from an as-grown sample. Interference fringes in the spectrum of the as-grown sample indicate Fabry-Perot modes due to vertical optical confinement. Majority of the photons generated by the MQWs remain trapped within the wafer, ${ }^{16}$ forming standing waves which are subsequently reabsorbed. Such oscillations were clearly suppressed in the nanopillar samples with diameters of 130,140,150, and $160 \mathrm{~nm}$ due to diffraction of the guided modes by the $\mathrm{PhC}$, together with the observation of significant PL intensity enhancements, demonstrating that $\mathrm{PhCs}$ can indeed play a remarkable role in manipulating spontaneous emission by suppressing unwanted optical modes via the PBG. Compared to the as-grown sample with peak emission wavelength at $455 \mathrm{~nm}$, the peak emission wavelengths from the nanopillar samples were centered at $443.79,444.05,446.84$, and $448.46 \mathrm{~nm}$, a systematic blueshift with respect to their diameters, which can be attributed to the position of the PBG. The spectral shifts were consequential of the overlap between the MQW emission band and the PBG. With decreasing pillar diameters, the position of the PBG was shifted from $490.59 \mathrm{~nm}$ to $431.96 \mathrm{~nm}$. As a result, the spectra contents in the shorter wavelength region were enhanced to a greater extent, giving rise to an apparent spectral blueshift. For the $140 \mathrm{~nm}$ diameter nanopillar array, a fourfold increase in PL intensity was observed since the position of the PBG coincided with the emission wavelength, as evidenced through the computed TE and TM band structures in Fig. 9. The TE band gap (the TE mode dominates in InGaN/GaN MQW LEDs) occurs in the frequency range between a/ $\lambda$ of 0.4211-0.4402 within the light line of air, corresponding to wavelengths between 436.17 and $455.95 \mathrm{~nm}$. It indicates the PBG band corresponding to the $140 \mathrm{~nm}$ nanopillar array overlaps optimally with the MQW emission band, correlating well with PL measurements. 
(a)

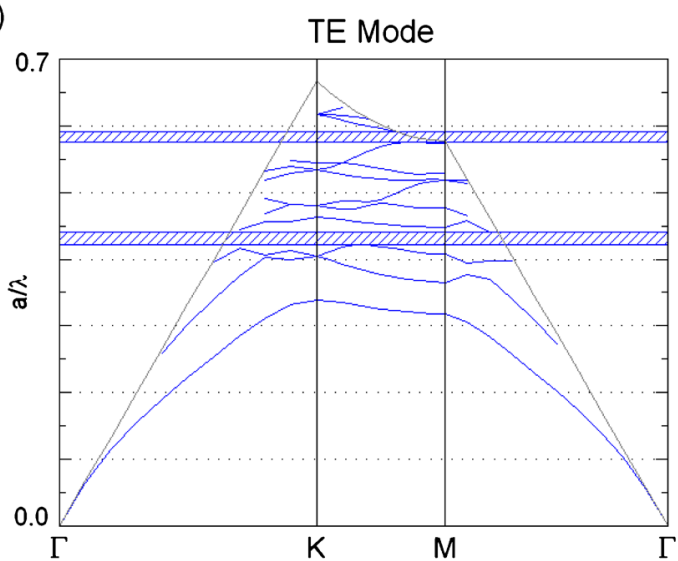

(b)

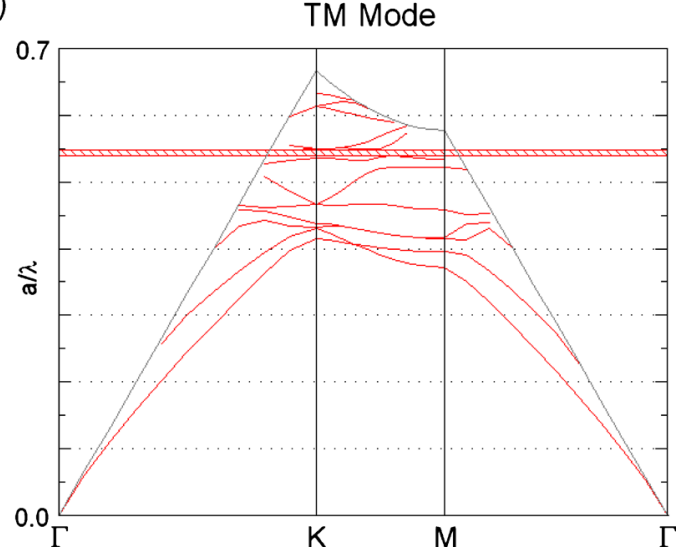

FIG. 9. (Color online) Computed (a) TE and (b) TM band structures for an air-spaced nanopillar array with pillar diameter and pitch of $140 \mathrm{~nm}$ and 192 $\mathrm{nm}$, respectively.

\section{The effects of disordering in nanopillar arrays}

With the technique of NSL, the formation of closedpacked hexagonal arrays relies on the packing and dimensions of self-assembling microspheres. To achieve a closepacked monolayer array by spin-coating, the sphere diffusion rate and concentration of the suspension play important roles in determining the coverage area. The former factor involves a balance between centrifugal forces controlled by rotation speed and surface tension forces which can be reduced by adding the surfactant SDS. Nevertheless, the presence of defects including point defects, line defects, and nonuniformity of sphere diameters are inevitable. Point and line defects are naturally and randomly formed during the self-assembly process. These defects are then transferred to the GaN wafer during etching, affecting the optical properties of the $\mathrm{PhC}$, which is investigated and reported in this section.

The nanospheres were shrunk with a $\mathrm{CHF}_{3}$-based etch recipe which targets $\mathrm{SiO}_{2}$. Figure 10 plots the diameters of nanospheres as a function of etch durations. The etching process also induces dimensional nonuniformity among spheres. Initially the $192 \mathrm{~nm}$ diameter spheres self-assemble into a monolayer with high uniformity $( \pm 2 \mathrm{~nm})$. Once the etch duration exceeds the seventh minute, the sphere shrinks rapidly. The rate of shrinkage increases further after the twentyfifth minute. Nevertheless, with increasing etching duration, the variation in diameters between spheres gradually en-

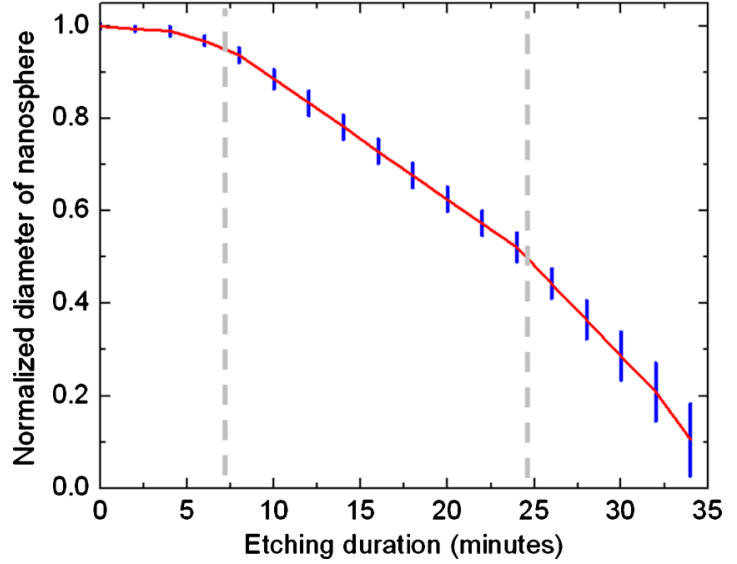

FIG. 10. (Color online) Plot of normalized diameter of nanosphere vs etch duration.

larges. When the diameters of spheres are below one-third of their original values, the variation becomes significant and causes poor uniformity $( \pm 8)$, as shown in Fig. 11. Therefore, there is a limit on the extent of shrinkage that can be tolerated. When the pattern is transferred to the GaN wafer, nanopillar arrays with poor uniformity of diameters are unfavorable for establishing a well-defined PBG.

To illustrate the effect of nonuniformity qualitatively, finite-difference time-domain (FDTD) simulations were carried out to predict the field distribution when a continuous wave at $440 \mathrm{~nm}$ was emitted from the center bottom position from arrays of nanopillars with different packing orders. Figure 12(a) shows the simulation result for an ideal air-spaced nanopillar array with diameter/pitch/height of $140 \mathrm{~nm} / 192$ $\mathrm{nm} / 350 \mathrm{~nm}$, whereby lateral propagation of light is obviously suppressed; based on the simulated band diagram a PBG is indeed predicted between $\sim 436$ and $455 \mathrm{~nm}$, correlating well with the FDTD simulated results. However, the presence of defects and nonuniformities disrupts the orderliness of the $\mathrm{PhC}$. As a result, losses and scattering effects are superimposed upon the PBG, the degree of which depends on the extent of defects. A simulation was then performed on a disordered array of nanopillars whereby randomly selected pillars with height of $350 \mathrm{~nm}$ have been shifted from their equilibrium positions. At the same time, the diameters of nanopillars in the array range between 130 and $150 \mathrm{~nm}$; such a "defective" array bears close resemblance to an actual fab-

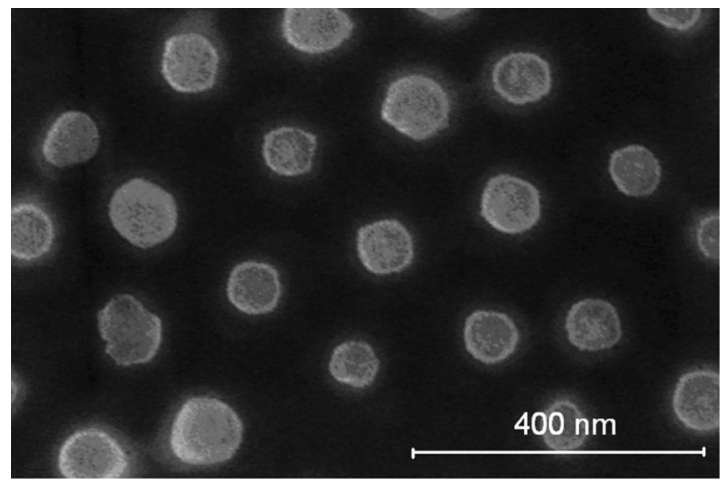

FIG. 11. FE-SEM image demonstrating poor dimensional uniformity of spheres due to excessive etching. 


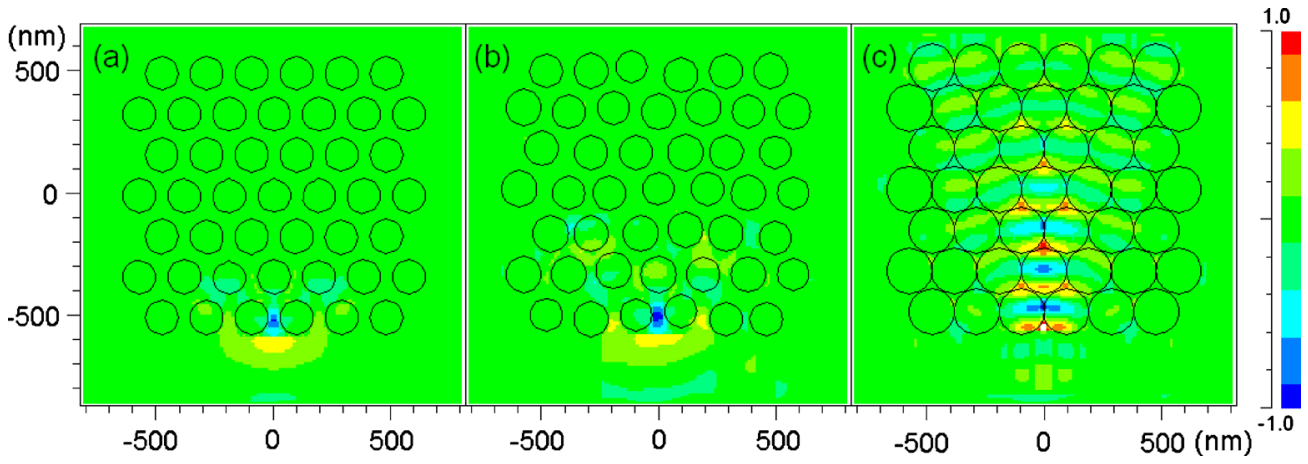

FIG. 12. (Color online) Computed FDTD results for (a) a perfect PhC structure comprising regular air-spaced nanopillars, (b) a PhC structure with defects, and (c) a close-packed nanopillar array.

ricated array under worst-case conditions. The results of this simulation are shown in Fig. 12(b). The introduced defects interrupt the periodic order causing some degree of scattering. The incident source is now partially reflected and partially transmitted by the array due to the presence of leakage modes. This also explains why the measured transmission dips are Gaussian in profile, instead of being abruptly sharp. To complete the picture, an FDTD simulation was also performed on a closed-pack $192 \mathrm{~nm}$ nanopillar array as illustrated in Fig. 12(c). The wave is allowed to propagate freely through the array in the absence of a PBG. Comparing the three presented scenarios, one can conclude that the airspaced nanopillar structure proposed in this work behaves as a leaky PhC, comprising a superposition of defect leakage modes upon PBG confinement modes. In spite of imperfections, its effectiveness on enhancing light extraction from $\mathrm{GaN}$ materials is well demonstrated, especially in consideration of the ease and cost of the self-assembled nanoscale patterning process.

\section{CONCLUSION}

In summary, the fabrication of ordered hexagonal array of air-spaced nanopillar on GaN wafers by NSL has been demonstrated. Employing a dual-step dry etch process, the dimensions of the nanopillars in an array can be adjusted without altering their pitch; at the same time, PBG properties of the self-assembled nanostructures can also be modified. The positions of the PBG were identified by optical transmission measurement, correlating well with the prediction of PWE stimulations. A maximum fourfold increase in PL intensity was observed compared to an as-grown sample, depending on the overlap between the PBG with the emission band. Despite the presence of leakage modes due to defects, we have demonstrated the effectiveness of the self- assembled PBG structures in suppressing unwanted guiding modes and promoting light extraction efficiency.

\section{ACKNOWLEDGMENTS}

This work was supported by a GRF grant of the Research Grant Council of Hong Kong (Project No. HKU 7118/ 09E).

${ }^{1}$ T. Fujii, Y. Gao, R. Sharma, E. L. Hu, S. P. DenBaars, and S. Nakamura, Appl. Phys. Lett. 84, 855 (2004).

${ }^{2}$ C. Y. Wang, L. Y. Chen, C. P. Chen, Y. W. Cheng, M. Y. Ke, M. Y. Hsieh, H. M. Wu, L. H. Peng, and J. J. Huang, Opt. Express 14, 10556 (2008).

${ }^{3}$ C.-C. Kao, H.-C. Kuo, H.-W. Huang, J.-T. Chu, Y.-C. Peng, Y.-L. Hsieh, C. Y. Luo, S.-C. Wang, C.-C. Yu, and C.-F. Lin, IEEE Photonics Technol. Lett. 17, 19 (2005)

${ }^{4}$ H. W. Choi, C. W. Jeon, C. Liu, I. M. Watson, M. D. Dawson, P. R. Edwards, R. W. Martin, S. Tripathy, and S. J. Chua, Appl. Phys. Lett. 86, 021101 (2005).

${ }^{5}$ S. X. Jin, J. Li, J. Y. Lin, and H. X. Jiang, Appl. Phys. Lett. 77, 3236 (2000).

${ }^{6}$ T. N. Oder, K. H. Kim, J. Y. Lin, and H. X. Jiang, Appl. Phys. Lett. 84, 466 (2004).

${ }^{7}$ K. J. Byeon, S. Y. Hwang, and H. Lee, Appl. Phys. Lett. 91, 091106 (2007).

${ }^{8}$ A. David, T. Fujii, R. Sharma, K. McGroddy, S. Nakamura, S. P. DenBaars, E. L. Hu, C. Weisbuch, and H. Benisty, Appl. Phys. Lett. 88, 061124 (2006).

${ }^{9}$ S. H. Kim, K. D. Lee, J. Y. Kim, M. K. Kwon, and S. J. Park, Nanotechnology 18, 055306 (2007).

${ }^{10}$ E. Yablonovitch, Phys. Rev. Lett. 58, 2059 (1987).

${ }^{11}$ J. C. Hulteen and R. P. Vanduyne, J. Vac. Sci. Technol. A 13, 1553 (1995).

${ }^{12}$ W. N. Ng, C. H. Leung, P. T. Lai, and H. W. Choi, Nanotechnology 19, 255302 (2008).

${ }^{13}$ W. Y. Fu, K. K. Y. Wong, and H. W. Choi, Appl. Phys. Lett. 95, 133125 (2009).

${ }^{14}$ P. G. Eliseev, G. A. Smolyakov, and M. Osinski, IEEE J. Sel. Top. Quantum Electron. 5, 771 (1999).

${ }^{15}$ M. Boroditsky, T. F. Krauss, R. Coccioli, R. Vrijen, R. Bhat, and E. Yablonovitch, Appl. Phys. Lett. 75, 1036 (1999).

${ }^{16}$ H. X. Jiang and J. Y. Lin, Crit. Rev. Solid State Mater. Sci. 28, 131 (2003). 